\title{
To the article
}

\section{"Dominant and Non-Dominant Frequency Structure of Evoked Ventricular Fibrillation in Dogs with Myocardial Ischemia" \\ by M. I. Guryanov, Vol. 161, No. 2, pp. 228-231, June, 2016}

The reference section for this paper should read

1. O. L. Bockeria and M. B. Biniashvili, Sudden cardiac death and ischemic heart disease. Ann. Aritmol., 10, No. 2, 69-79 (2013).

2. L. A. Bockeria, O. L. Bockeria, and O. N. Kislitsina, Randomized clinical trials for prevention of sudden cardiac death: principles and total scores. Ann. Aritmol., 7, No. 2, 5-14 (2010).

3. N. L. Gurvich, Basic Principles of Heart Defibrillation [in Russian], Moscow (1975).

4. M. I. Guryanov, Dominant and Non-Dominant Structure of Ventricular Fibrillation in Canine Heart. Bull. Exp. Biol. Med., 160, No. 3, 291-293 (2016).

5. V. N. Orlov, Manual for Electrocardiography [in Russian], Moscow (2012).

6. A. Amann, R. Tratnig, and K. Unterkofler, Reliability of old and new ventricular fibrillation detection algorithms for automated external defibrillators. Biomed. Eng. OnLine, 4, doi: 10.1186/1475-925X-4-60 (2005).

7. K. A. Cheng, D. J. Dosdall, L. Li, J. M. Rogers, R. E. Ideker, and J. Huang, Evolution of activation patterns during longduration ventricular fibrillation in pigs. Am. J. Physiol. Heart Circ. Physiol., 302, No. 4, H992-H1002 (2012).

8. J. J. Goldberger, A. Basu, R. Boineau, A. E. Buxton, M. E. Cain, J. M. Canty Jr, P. S. Chen, S. S. Chugh, O. Costantini, D. V. Exner, A. H. Kadish, B. Lee, D. Lloyd-Jones, A. J. Moss, R. J. Myerburg, J. E. Olgin, R. Passman, W. G. Stevenson, G. F. Tomaselli, W. Zareba, D. P. Zipes, and L. Zoloth, Risk stratification for sudden cardiac death: a plan for the future. Circulation, 129, No. 4, 516-526 (2014).

9. J. Huang, J. M. Rogers, C. R. Killingsworth, K. P. Singh, W. M. Smith, and R. E. Ideker, Evolution of activation pat- terns during long-duration ventricular fibrillation in dogs. Am. J. Physiol. Heart Circ. Physiol., 286, No. 3, H1193-H1200 (2004).

10. J. F. Huizar, M. D. Warren, A. G. Shvedko, J. Kalifa, J. Moreno, S. Mironov, J. Jalife, and A. V. Zaitsev, Three distinct phases of VF during global ischemia in the isolated bloodperfused pig heart. Am. J. Physiol. Heart Circ. Physiol., 293, No. 3, H1617-H1628 (2007).

11. L. Li, Q. Jin, D. J. Dosdall, J. Huang, S. M. Pogwizd, and R. E. Ideker, Activation becomes highly organized during long-duration ventricular fibrillation in canine hearts. Am. J. Physiol. Heart Circ. Physiol., 298, No. 6, H2046-H2053 (2010).

12. L. Li, X. Zheng, D. J. Dosdall, J. Huang, S. M. Pogwizd, and R. E. Ideker, Long-duration ventricular fibrillation exhibits 2 distinct organized states. Circ. Arrhythm. Electrophysiol., 6, No. 6, 1192-1199 (2013).

13. C. Lin, Q. Jin, N. Zhang, J. Zhou, Y. Pang, Y. Xin, S. Liu, $\mathrm{Q}$. $\mathrm{Wu}$, and $\mathrm{L}$. $\mathrm{Wu}$, Endocardial focal activation originating from Purkinje fibers plays a role in the maintenance of long duration ventricular fibrillation. Croat. Med. J., 55, No. 2, 121-127 (2014).

14. Z. Qu and J. N. Weiss, Mechanisms of ventricular arrhythmias: from molecular fluctuations to electrical turbulence. Annu. Rev. Physiol., 77, 29-55 (2015).

15. R. P. Robichaux, D. J. Dosdall, J. Osorio, N. W. Garner, L. Li, J. Huang, and R. E. Ideker, Periods of highly synchronous, non-reentrant endocardial activation cycles occur during long-duration ventricular fibrillation. J. Cardiovasc. Electrophysiol., 21, No. 11, 1266-1273 (2010). 